\title{
OPTIMIZED ENERGY EFFICIENT LIGHTING SYSTEM FOR GREEN BUILDINGS
}

\author{
Alisha Jadhav' ${ }^{1}$, D.M. Chandwadkar ${ }^{2}$ \\ ${ }^{1,2}$ Department of Electronics and Telecommunication, KK Wagh Institute of Engineering Education and Research, \\ Nashik, Pune University \\ 1'alisha.0511@gmail.com, ${ }^{2}$ dmchandwadkar@kkwagh.edu.in
}

\begin{abstract}
Increasing population places increasing demands on energy supply, which leads to heavy power deficiency. We can supply only a limited amount of energy to the consumers because we have only limited conventional sources of energy and non-conventional energy sources are mostly still under research and have not been deployed on a large scale. Not only there is limited amount of energy to be supplied to the consumers but heavy demand of energy supply also leads to environment degradation. This creates a need to use the limited energy efficiently. This has given rise to new trend towards reducing the energy consumption: Green Buildings. About $40 \%$ of the energy consumption in the world is consumed by buildings [1]. Energy consumed by lighting systems accounts for about 15\% of total energy consumption of buildings [1]. Thus we need to use energy efficient lighting devices for illuminating buildings. Thus in this study we to tend to develop optimized energy efficient system, which will use LED lights to illuminate buildings. The illumination intensity of the LED lights will be decided by the FPGA, which is interfaced to the LED driver. Based on combined values of light intensity of the room and occupancy of the room using different sensors, the light output of the LED will be controlled.
\end{abstract}

Keywords: LED Lighting System, FPGA, Green Buildings, Auto-dimming.

\section{INTRODUCTION}

Deficiency of power is becoming a serious worldwide issue. Available energy sources must be used efficiently; unnecessary wastage of power be brought down. Inefficient lighting systems of buildings account for large fraction of the electricity consumption in the world. About $40 \%$ of the energy consumption in the world is consumed by buildings. Energy consumed by lighting systems accounts for about $15 \%$ of total energy consumption of buildings [1]. Buildings account for $8 \%$ of the total country's utilization in terms of power and grow steadily at 11 to $12 \%$ per annum. This rate of growth of energy demand among buildings is much higher than the $6 \%$ growth that the electricity sector sees overall [2]. The reason behind such wastage of energy in buildings is that most of buildings today are over-lit because conventional lighting systems have only two levels of brightness; either the lights are operated at full power when turned on or zero power when turned off. Lights are on even in the presence of daylight and when spaces are unoccupied, also sometimes we forget to turn off the lights. Lack of dimmable lighting systems not only wastes energy but also creates discomfort to the dwellers which results in reduced productivity.

Energy produced by depleting natural resources as well as causing $\mathrm{CO} 2$ emissions is critical environmental impacts. Volatile energy markets, rising energy costs and increasing environmental awareness about issues such as global warming make energy efficiency and conservation a high priority [3]. Buildings and the infrastructure available to the people should be made green in the sense that they use resources more efficiently and at the same time are environmental friendly. A green building is an environmentally sustainable building, designed, constructed and operated to minimize the total environmental impacts [3].

Because of uncontrolled wastage of energy by the existing inefficient illumination systems of buildings there is urgent need of energy efficient lighting device as well as adaptable illumination system. Over the years LED (Light Emitting Diode) lights have emerged as a green lighting source. LED lights basically use a number of LEDs connected either in series or in parallel. LED average life expectancy of 10 million hours, security and reliability strong, non-glare, nonmercury, sodium and other elements of potential health hazards of the material is conducive to environmental protection, known as the "Green Lighting source" [4]. Distinguishing features of LED lights such as energy efficiency, environmental friendly, longer operational life, smaller size, have made it the first choice as an illumination source to the lighting system designers.

LED lights are different from other light sources w.r.t three parameters which are source of light, directionality, heat. An electrical current passes through semiconductor material, which illuminates the tiny light sources we call LEDs. The heat produced is absorbed into a heat sink [6]. LED lights emit in a specific direction. According to repeatable experiments, LED can save at least $80 \%$ of electricity than incandescent lamps, and at least $50 \%$ of electricity than fluorescent lamps for same level of illumination. Moreover, a LED lamps working life is 20 times longer than an incandescent lamp, and twice as longer as fluorescent lamp [5]. 
In this paper, we proposed a dimmable LED lighting system for buildings which adapts to the ambient light creating an optimised energy efficient lighting system. The dimming of light is done by a control unit based on decision made by processing the sensor data in real-time. Brightness of the LED lights is adjusted depending on the ambient light intensity and occupancy of the space.

\section{RELATED WORK}

In [4] an intelligent dimming control system based on LED lamps is presented. The system has two working modes; manual and automatic mode. A STC-MCU (Micro Controller Unit) is used to process the information and generate PWM control signals to set the LED lamps into predefined brightness levels. In [5] proposed an intelligent control system for LED nightlight by PWM (Pulse Width Modulation) dimming to change the light intensity real-time using a microcontroller. Moonlight simulation helps to give sound sleep. In [7] an ARM 7 based embedded system design with a day light and a PIR (Pyroelectric Infrared) sensor is developed to control the light intensity in a household environment. In [8] a wireless network enabled LED dimming system with an android remote control is proposed in which microcontroller generates the PWM dimming signal to adjust the brightness of the LEDs. In [9] an STC-MCU is used to process the motion sensor information and generate PWM signals to adjust the brightness of the white LED and yellow LED respectively. In [10] presented a wireless embedded control which can either switch on/off the lights or reduce the light intensity of LED light for indoor as well as outdoor lighting, reducing the power consumption drastically. ATMEGA32, 8-bit microcontroller is used to generate PWM waves to control the light intensity of the LED light. In [11] proposes a low cost, wireless, optimized smart LED lighting system which automatically adjust the light intensity which reduces energy consumption while maintaining user satisfaction. MSP430 family of ultra low power microcontrollers are used in the control units.

As we can see that all of the work done in the field of designing energy efficient lighting system till date has used microcontrollers to generate control signal to the adjust the brightness of the LED lights using different light and motion detection sensors data in real-time. No one has used a reconfigurable control unit yet. Use of microcontrollers creates a software solution which has lower speed of operation and also the performance is low, not much energy efficient.

In contrast to the use of microcontrollers, in this paper we have proposed an optimized energy efficient lighting control system using FPGA (Field Programmable Gate Array) as the main control unit which does the sensor data processing and generation of brightness levels to adjust the brightness of the LED lights as per the ambient light. We are all familiar with low cost low power consumption features of FPGA. At the same it is parallel in processing and gives high performance. Thus use of an FPGA in system creates a complete hardware module.

\section{SYSTEM DESIGN}

\section{A. Sensor unit}

The basic principle of operation of the system lies in data collection and processing. The system mainly consists of three parts: the sensor unit, control unit and the LED lights and necessary circuirtry.

1) Motion detector: Here a Pyroelectric infrared (PIR) sensor detects the presence of a person inside a room. The basic principle of operation of PIR motion is that it detects the infrared rays emitted from human or animals and its output is an electrical signal.

2) Light sensor: Detects the light intensity of the room for indoor lighting.

3) Analogue-to-digital converter: FPGA pins require digital signals and sensors produce analog signals. Thus for compatibility, ADC is used to the necessary signal conversion between FPGA and sensors. ADC with I2C compatible serial interface is chosen for the system which is I2C slave.

\section{B. Control unit}

In this proposed system a field programmable array (FPGA) works as the control unit. Peripherals are interfaced to the control unit through an $\mathrm{I} 2 \mathrm{C}$ serial bus. I2C protocol is chosen for communication because it is very easy to implement, it uses only two wires serial data (SDA) and serial clock (SCL). I2C bus master is implemented on the FPGA itself. Control unit takes the digital value of the sensor data from the ADC and generates binary value according to the algorithm of dimming the LEDs lights. Algorithm is implemented as a finite state machine on the FPGA.

\section{LED circuitry}

It consists of LED lights and LED driver circuit. LED driver is interfaced to the control unit as I2C bus slave. LED driver takes binary values from the control unit to set the brightness level of the LED light. It converts the binary values to respective PWM signal for adjusting the light intensity of the LED lights.

\section{Proposed System}

Fig. 1 shows the block schematic of the proposed optimized energy efficient lighting system for green buildings. LED lights will be turned on only if motion of user is detected inside the room. If motion is detected then light sensor will detect the light intensity of the room. And depending on the light intensity detected respective brightness level will be generated in binary which will be sent to the LED driver which in turn will do the processing and send PWM signals to the LED lights. The LED lights will glow at the set brightness level. This process of motion detection, sensing of ambient light and auto adjusting of LED light brightness works in a continuous loop giving an energy efficient, comfortable working environment to the user. 


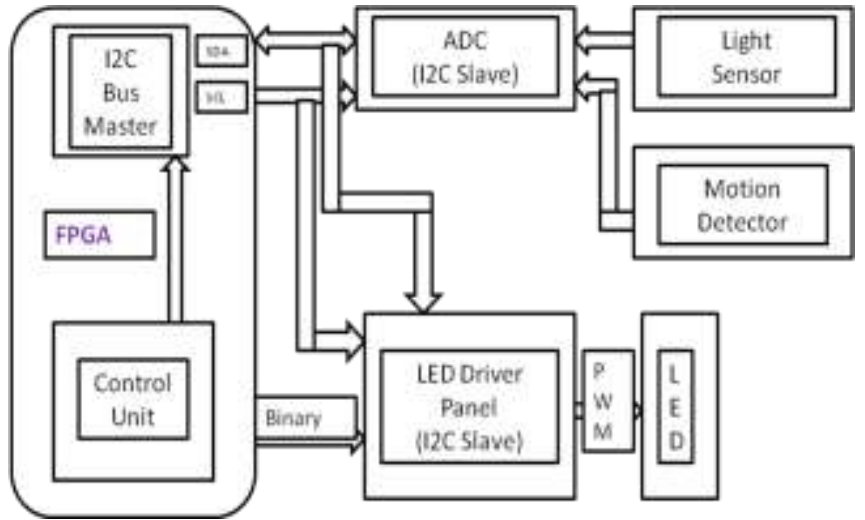

Fig.1. Block schematic of the proposed optimized energy efficient lighting system for green buildings.

\section{E. Flow chart}

A generalized flow chart for intelligent auto-dimming of LED lights is shown in Fig.2. According the requirement of user different threshold levels could be set for light intensity depending on the time of the day and respective binary value for adjusting brightness of LED could be set. Proper delays would be set to prevent flickering of light.

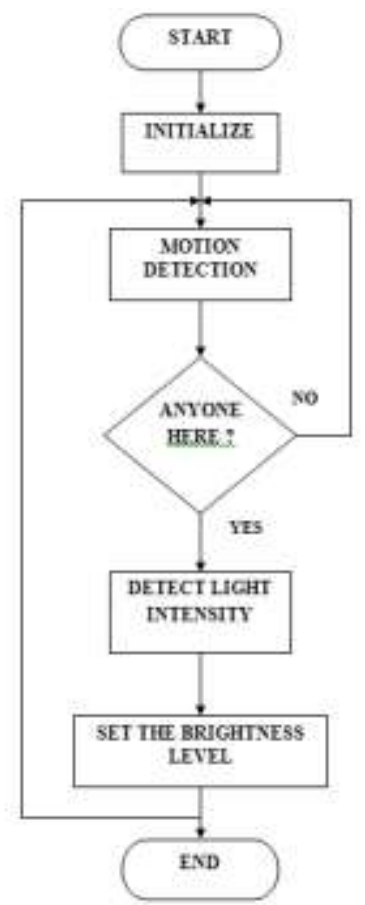

Fig. 2. Generalized Flow chart.

\section{CONCLUSIONS}

Thus we can summarize the work; we have proposed an energy efficient lighting control system for green buildings. To achieve energy efficiency we used energy efficient lighting source such as LED lamps. The illumination of the room is set according to the ambient light intensity detected or else if no one is present in the room lights remains off. Use of FPGA as the control unit rather than using microcontrollers create a complete hardware module which is faster in speed due to parallel computation and also the control algorithm can be modified on the fly as per users requirements, provides larger number of input/output ports.

Application area includes: apartments, Office buildings of the corridor lights, bathroom lighting, hotel lobby, malls. In these above mentioned places the proposed system can achieve very good lighting, energy saving, environmental protection effect. As due to no provision for dimming of lights in these places a lot of energy is wasted.

\section{FUTURE SCOPE}

The devices connected to the FPGA can use either a wired connection or a wireless one. In this proposed system wired solutions were used, however, the interface can be easily replaced by a wireless solution. A wireless network approach can be integrated to the lighting control system which is proposed in this paper, which will monitor and control the whole building, creating Green Buildings.

\section{ACKNOWLEDGMENT}

The authors would like to acknowledge the support of K.K.Wagh Institute of Engineering Education Research, Nashik, India for providing research facilities.

\section{REFERENCES}

[1] Sunghoi Park; Soono Seo; Byunghoon Lee; Jinsung Byun; Sehyun Park, "An energy efficient smart LED lighting system for building energy management," in Consumer Electronics (ISCE 2014), The 18th IEEE International Symposium on , vol., no., pp.1-2, 22-25 June 2014

[2] http://www.thealternative.in/lifestyle/10-greenbuilding-innovations-in-india

[3] http://buildgreen.co.nz/definition.html

[4] Jia Dongying; Wang Wei, "The intelligent system for LED lighting based on STCMCU," in Computer and Communication Technologies in Agriculture Engineering (CCTAE), 2010 International Conference On , vol.3, no., pp.445-447, 12-13 June 2010

[5] Shuai Han; Xiao Zhong; Yifeng Ding; Wei Li; Song Liu; Peng Liu, "Intelligent Dimming LED for Moonlight Simulation," in Information Science and Control Engineering (ICISCE), 2015 2nd International Conference on , vol., no., pp.778-782, 24-26 April 2015

[6] https://www.energystar.gov/products/

[7] Sulthana, M.; Rao, N.U., "An ARM-7 based embedded energy efficient LED lighting system for domestic applications with user satisfaction," in Wireless and Optical Communications Networks (WOCN), 2014 Eleventh International Conference on , vol., no., pp.15, 11-13 Sept. 2014

[8] Kok-Hua Teng; Zi-Yi Lam; Sew-Kin Wong, "Dimmable WiFi-connected LED driver with android based remote control," in Wireless Technology and Applications (ISWTA), 2013 IEEE Symposium on , vol., no., pp.306-309, 22-25 Sept. 2013

[9] Qi Chunhui; Wang Wei, "A LED Control System with Brightness and Color Temperature Adjustable," in Control, Automation and Systems Engineering (CASE), 
2011 International Conference on , vol., no., pp.1-4, 30-31 July 2011

[10] Thattai, K.; Manikanta, K.B.; Chhawchharia, S.; Marimuthu, R., "ZigBee and ATmega32 based wireless digital control and monitoring system For LED lighting," in Information Communication and Embedded Systems (ICICES), 2013 International Conference on , vol., no., pp.878-881, 21-22 Feb. 2013

[11] Magno, M.; Polonelli, T.; Benini, L.; Popovici, E., "A Low Cost, Highly Scalable Wireless Sensor Network Solution to Achieve Smart LED Light Control for Green Buildings," in Sensors Journal, IEEE , vol.15, no.5, pp.2963-2973, May 2015. 